\title{
On a conjecture of W. Veys
}

\author{
E. Artal Bartolo $\cdot$ I. Luengo $•$ A. Melle-Hernández
}

\begin{abstract}
In this work we show a singular $K 3$ surface $S$ of $\mathbb{P}^{3}$ such that $\chi\left(\mathbb{P}^{3} \backslash S\right)=0$ producing a counter-example to a conjecture of Veys.

Mathematics Subject Classification (1991): 14J26, 14E07

In this journal W. Veys [V, p. 547] stated the following

Conjecture. Let $C_{i}, 1 \leq i \leq r$, be irreducible hypersurfaces in $\mathbb{P}^{n}(\mathbb{C})$. If

$$
(-1)^{n} \chi\left(\mathbb{P}^{n} \backslash \bigcup_{i=1}^{r} C_{i}\right) \leq 0
$$
\end{abstract}

then all $C_{i}$ have Kodaira dimension $-\infty$.

This conjecture was proven for $n=2$ by A.J. de Jong and J. Steenbrink in [JS]. Also, R. Gurjar and A. Parameswaran [GP] gave an independent proof. In this note we give a counterexample to this conjecture for $n=3$.

As W. Veys has pointed out to us, this problem is related with the monodromy conjecture of the topological and Igusa zeta-functions of singularities of hypersurface, see [V].

Counterexam ple to the conjecture of Veys. Let $D \subset \mathbb{P}^{2}$ be a reduced projective plane curve of degree $d$, with singularities $\operatorname{Sing}(D)=\left\{P_{1}, \ldots, P_{s}\right\}$. The Euler characteristic of $D$ is given by the well-known formula

$$
\chi(D)=3 d-d^{2}+\sum_{i=1}^{s} \mu\left(D, P_{i}\right),
$$

E. Artal Bartolo

Departamento de Matemáticas, Universidad de Zaragoza, Campus Plaza San Francisco s/n, E-50009 Zaragoza, Spain (e-mail: artal@posta.unizar.es)

I. Luengo, A. Melle-HernándeZ

Departamento de Álgebra, Universidad Complutense, Ciudad Universitaria s/n, E-28040 Madrid, Spain (e-mail: \{iluengo,amelle\}@eucmos.sim.ucm.es)

First author was partially supported by DGES PB97-0284-C02-02; last two authors were partially supported by DGES PB97-0284-C02-01 
where $\mu\left(D, P_{i}\right)$ is the Milnor number of the curve $D$ at $P_{i}$. Recall that a simple singularity of type $\mathbb{A}_{n}$ means a singularity locally defined by $v^{2}+u^{n+1}=0$, and so it has Milnor number equal to $n$.

Let $C_{1}$ and $C_{2}$ be the projective plane curves of degree 3 in $\mathbb{P}^{2}$ given as the zero locus of the homogeneous polynomials $f_{1}(x, y, z):=x^{3}+y^{2} z-3 x^{2} z$ and $f_{2}(x, y, z):=x^{3}+y^{2} z-3 x^{2} z+4 z^{3}$. Both cubics have only one singular point, which is of type $\mathbb{A}_{1} ;$ choosing as above homogeneous coordinates $(x: y: z)$ in $\mathbb{P}^{2}$ the curve $C_{1}$ is singular at $P_{1}=(0: 0: 1)$ and $C_{2}$ is singular at $P_{2}=(2: 0: 1)$. Then $\chi\left(C_{1}\right)=\chi\left(C_{2}\right)=1$.

Let $C=C_{1} \cup C_{2}$ be the corresponding sextic which has three singular points, namely $P_{1}, P_{2}$ and $P_{3}=C_{1} \cap C_{2}=(0: 1: 0)$; also $C$ has at $P_{3}$ a simple singularity of type $\mathbb{A}_{17}$. It means again that the Euler characteristic $\chi(C)=1$.

Let $X$ be the normal compact complex surface obtained as a double covering of $\mathbb{P}^{2}$ branched along $C$. The surface $X$ has only a finite (three) number of rational singularities. Let $\sigma: \widetilde{X} \rightarrow X$ be the canonical resolution of these singularities. Then $\widetilde{X}$ is the minimal resolution of the double covering of the projective plane ramified on a sextic curve having only simple singularities: $\widetilde{X}$ is a $K 3$ surface, see [BPV, pp. 182-183]. Then the Kodaira dimension $\kappa(\widetilde{X})$ of $\widetilde{X}$ is 0 . Since the Kodaira dimension is a birational invariant, $\kappa(X)=0$ (e.g. see [H, p. 421]).

The idea is to find a surface $S \subset \mathbb{P}^{3}$ birationally equivalent to $X$ such that $\chi\left(\mathbb{P}^{3} \backslash S\right)=0$, or, equivalently, $\chi(S)=4$. This last equivalence is a consequence of:

Additivity Principle. Let $Y$ be an analytic variety. Let $\left\{S_{i}\right\}_{i \in I}$ be a finite prestratification of $Y$, i.e., $I$ is a finite set, $Y=\coprod_{i \in I} S_{i}$ and $S_{i}$ are locally closed analytic subvarieties of $Y$ for $i \in I$. Then

$$
\chi(Y)=\sum_{i \in I} \chi\left(S_{i}\right)
$$

Let $S \subset \mathbb{P}^{3}$ be the irreducible surface given by the zero locus of the homogeneous polynomial of degree 6

$$
\begin{aligned}
f_{6}(x, y, z) & =f_{1}(x, y, z) f_{2}(x, y, z)+w^{2} z^{4} \\
& =\left(x^{3}+y^{2} z-3 x^{2} z\right)\left(x^{3}+y^{2} z-3 x^{2} z+4 z^{3}\right)+z^{4} w^{2} .
\end{aligned}
$$

It can be easily seen that $S$ and $X$ are birationally equivalent: Take the affine chart $(x, y)$ of $\mathbb{P}^{2}$; the affine part $A$ of $C$ is the disjoint union of two nodal cubics with only one (common) place at infinity each one; the equation of $A$ is $f_{1}(x, y, 1) f_{2}(x, y, 1)=0$; therefore, $\chi(A)=0$. Take the double covering $\breve{S}$ of $\mathbb{C}^{2}$ ramified on $A$. If we denote by $f(x, y)=0$ the equation of $A$, then $S$ is defined in $\mathbb{C}^{3}$ with coordinates $(x, y, w)$ as $f_{1}(x, y, 1) f_{2}(x, y, 1)+w^{2}=0$. We obtain that $X$ is birationally equivalent to $\breve{S}$, because of the birational equivalence of $\mathbb{C}^{2}$ and $\mathbb{P}^{2} ; S$ is the hypersurface of $\mathbb{P}^{3}$ defined by the homogeneisation of 
$f_{1}(x, y, 1) f_{2}(x, y, 1)+w^{2}$ in $z$, and as $\breve{S}$ is birationally equivalent to $S$ then $\kappa(S)=0$.

Let us compute $\chi(S)$ with the help of the following partition of $S$ into two disjoint parts: $S_{1}:=S \cap\{z=0\}$ and $S_{2}:=S \cap\{z \neq 0\}$.

Since $S_{1}=\{x=z=0\} \cong \mathbb{P}^{1}$, we have $\chi\left(S_{1}\right)=2$.

We can identify $S_{2}$ with $\breve{S}$. Let us consider the double covering $\pi: \check{S} \rightarrow$ $\mathbb{C}^{2}$, defined by $(x, y, w) \mapsto(x, y)$. Recall that $\pi_{\mid \pi^{-1}(A)} \pi^{-1}(A) \rightarrow A$ is an isomorphism and $\pi_{\mid \breve{S} \backslash \pi^{-1}(A)} \check{S} \backslash \pi^{-1}(A) \rightarrow \mathbb{C}^{2} \backslash A$ is an unramified double covering. Then by the standard properties of coverings, the Additivity Principle and the fact that $\chi(A)=0$ :

$$
\begin{aligned}
\chi\left(S_{2}\right) & =\chi(\check{S})=\chi\left(\check{S} \backslash \pi^{-1}(A)\right)+\chi\left(\pi^{-1}(A)\right)=2 \chi\left(\mathbb{C}^{2} \backslash A\right)+\chi(A) \\
& =2 \cdot 1+0=2 .
\end{aligned}
$$

In particular, $\chi(S)=4$.

We have found in this way several other examples of surfaces disproving the conjecture of Veys, this one being the simplest. In all cases, the Kodaira dimension $\kappa\left(C_{i}\right)$ of each irreducible component is zero. It will be interesting to know which is the upper bound for this Kodaira dimension $\kappa\left(C_{i}\right)$ under the topological hypothesis of the Euler characteristic of the complement verifying

$$
(-1)^{n} \chi\left(\mathbb{P}^{n} \backslash \bigcup_{i=1}^{r} C_{i}\right) \leq 0
$$

\section{References}

[BPV] W. Barth, C. Peters and A. Van de Ven, Compact complex surfaces, Erg. der Math. und ihrer Grenz., A Series of Modern Surveys in Math., 3, 4, Springer-Verlag, Berlin, 1984

[GP] R. Gurjar and A. Parameswaran, Open surfaces with non-positive Euler characteristic, Compositio Math. 99 (1995), 213-229

[H] R. Hartshorne, Algebraic Geometry, Graduate Texts in Math. 52, Springer-Verlag, New York, 1977

[JS] A.J. de Jong and J. Steenbrink, Proof of a conjecture of W. Veys, Indag. Math. 6 (1) (1995), 99-104

[V] W. Veys, Structure of rational open surfaces with non-positive Euler characteristic, Math. Ann. 312 (1998), 527-548 